\title{
Terahertz quantum cascade lasers with quasi-periodic resonators
}

\author{
Lukas Mahler, Alessandro Tredicucci, \\ Richard P. Green and Fabio Beltram \\ NEST/INFM and Scuola Normale Superiore, \\ Piazza dei Cavalieri 7, \\ Pisa, I-56126, Italy \\ email: L.Mahler@sns.it
}

\author{
Harvey E. Beere and David A. Ritchie \\ Cavendish Laboratory, \\ University of Cambridge, \\ Madingley Road, \\ Cambridge CB3 OHE, \\ United Kingdom
}

\begin{abstract}
We report simulations and experimental results of THz QCLs with quasi-periodic resonators based on a Fibonacci sequence.

Terahertz quantum cascade lasers were first demonstrated in 2002 [1] and have since undergone rapid development. One of the most active areas of reasearch into these devices has been the development of devices emitting in a single longitudinal mode through the use of distributed feedback (DFB) resonators. Because the optical mode in these devices arises through a surface plasmon, it is possible to implement them with periodically arranged slits in the top metallisation. These have been demonstrated for both single- and doublemetal waveguides [2],[3],[4].
\end{abstract}

Since the patterns are easily defined with optical contact lithography, this system is well suited to study novel resonator concepts like quasi-periodic or random structures. As a first step, a Fibonacci structure was chosen. It is usually generated by two different layers of dielectric A and B stacked according to the following rule: $S_{j+1}=\left\{S_{j-1}, S_{j}\right\}$ for $j>1$, with $S_{0}=\{B\}$ and $S_{1}=\{A\}$. A sequence of this type shows interesting transport properties for light [5]. Like in a DFB, a range of forbidden energy occurs, a so-called pseudouse of Distributed feed band gap, where light transport is prohibited and the wavefunctions decay exponentially. The existence of a pseudo-gap and the related peak in the density of states make this configuration interesting for alternative DFB resonators. The degeneracy between the two band-edges is removed, and improved stability against variations in the periodicity is found. In order to fabricate such a resonator as a THz-DFB, a slit was introduced each time the material would change from A to B. Thus the reflection at a dielectric interface is replaced by the reflection at the narrow barrier for the surface plasmon (Fig. 1(c)). Fig. 1(a) shows a measured spectrum of a $2.5 \mathrm{THz}$ QCL with a quasi-period of $6.4 \mu \mathrm{m}$; in devices with 6.2 and $6.6 \mu \mathrm{m}$ gratings no lasing occurred, most likely due to the band-edge of the pseudo-gap being detuned from the gain. Improved results should be achievable in double-metal waveguides; in fact the numerical simulations shown in Fig. 1(b) indicate a much clearer resonance at the band-edge in this case.
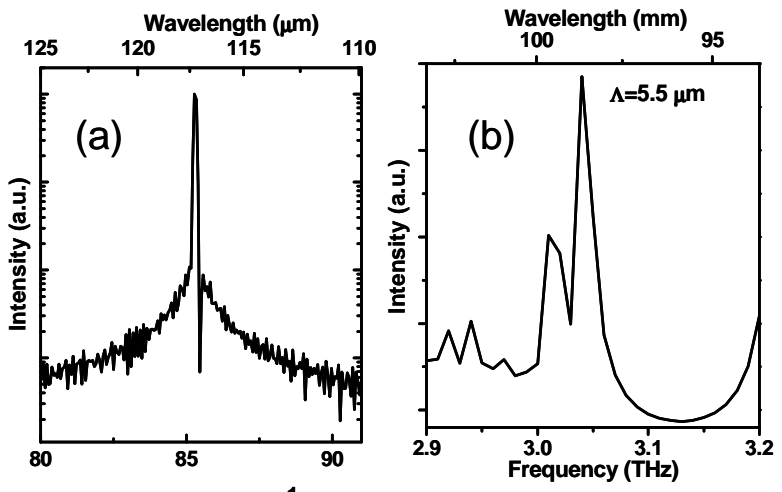

Wavenumber $\left(\mathrm{cm}^{-1}\right)$

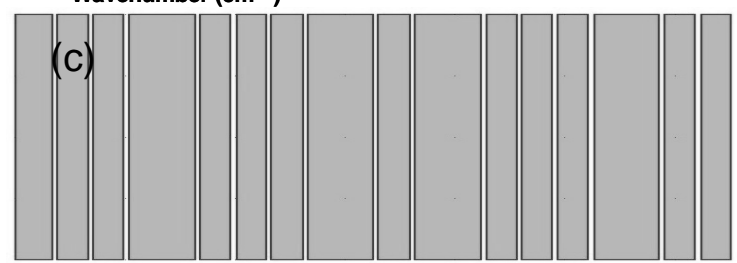

Fig. 1. (a) Laser emission spectrum measured from a single plasmon $\mathrm{THz}$ QCL with a Fibonacci resonator. (b) Calculated maximum intensity for a similar device with a double metal waveguide, excited with a point source. (c) Schematic diagram of the structure used.

\section{REFERENCES}

[1] R. Köhler, A. Tredicucci, F. Beltram, H. Beere, E. Linfield, A. Davies, D. Ritchie, R. Iotti, and F. Rossi, "Terahertz semiconductor heterostructure laser," Nature, vol. 264, no. 1, p. 417, May 2002.

[2] L. Mahler, A. Tredicucci, R. Köhler, F. Beltram, H. E. Beere, E. H. Linfield, and D. A. Ritchie, "High performance operation of single mode terahertz quantum cascade lasers with metallic gratings," Applied Physics Letters, vol. 87, no. 18, p. 181101, 2005.

[3] O. Demichel, L. Mahler, T. Losco, C. Mauro, R. Green, J. Xu, A. Tredicucci, F. Beltram, H. E. Beere, D. A. Ritchie, and V. Tamošiūnas, "Surface plasmon photonic structures in terahertz quantum cascade lasers," Optics Express, vol. 14, no. 12, pp. 5335-5345, 2006.

[4] J. A. Fan, M. A. Belkin, F. Capasso, S. Khanna, M. Lachab, A. G. Davies, and E. H. Linfield, "Surface emitting terahertz quantum cascade laser with a double-metal waveguide," Optics Express, vol. 14, no. 24, pp. 11672 $11680,2006$.

[5] L. Dal Negro, C. J. Oton, Z. Gaburro, L. Pavesi, P. Johnson, A. Lagendijk, R. Righini, M. Colocci, and D. S. Wiersma, "Light transport through the band-edge states of fibonacci quasicrystals," Phys. Rev. Lett., vol. 90, no. 5, p. 055501, Feb 2003. 